\title{
A CMOS-3D Reconfigurable Architecture with In-pixel Processing for Feature Detectors
}

\author{
M. Suárez, V.M. Brea, F. Pardo \\ Centro de Investigación en Tecnologías de la Información \\ (CITIUS) \\ University of Santiago de Compostela \\ Santiago de Compostela, Spain \\ Email:manuel.suarez.cambre@usc.es
}

\author{
R. Carmona-Galán \\ A. Rodríguez-Vázquez \\ Instituto de Microelectrónica de Sevilla (IMSE-CNM) \\ CSIC \\ Universidad de Sevilla \\ Sevilla, Spain
}

\begin{abstract}
This paper introduces a two-tier CMOS-3D architecture for generation of Gaussian pyramids, detection of extrema, and calculation of spatial derivatives in an image. Such tasks are included in modern feature detectors, which in turn can be used for operations like object detection, image registration or tracking. The top tier of the architecture contains the image acquisition circuits in an array of $320 \times 240$ active photodiode sensors (APS) driving a smaller array of $160 \times 120$ analog processors for low-level image processing. The top tier comprises in-pixel Correlated Double Sampling (CDS), a switched-capacitor network for Gaussian pyramid generation, analog memories and a comparator for in-pixel Analog to Digital Converter (ADC). The reuse of circuits for different functions permits to have a small area for every pixel. The bottom tier of the architecture contains a frame buffer with a set of registers acting as a frame-buffer with a one-to-one correspondence with the analog processors in the top tier, the digital circuitry necessary for the extrema detection and the calculation of the first and second spatial derivatives in the image, as well as Harris and Hessian point detectors. For the time being, a behavioral model of the first tier including mismatch and feedthrough and charge injection errors is discussed. Also, a VHDL model for the bottom tier is addressed. The two-tier architecture is conceived for its implementation on the $130 \mathbf{~ n m}$ CMOS-3D technology from Tezzaron. A companion chip will perform the higher-level operations as well as communications. In this technology an area of $\mathbf{3 0 0} \mu^{2}$ per analog processor has been estimated. The architecture proposed for pyramid generation lets a frame rate of 180 frames/s for an ADC conversion time of 120 $\mu s$. The architecture has been proved with object detection for a given feature detector.
\end{abstract}

\section{INTRODUCTION}

Operations as object detection and recognition [1], image retrieval, image registration, or tracking rely on local properties. Feature detectors as Harris [2], Harris-affine [3], Hessian [4], Hessian-affine [5], Scale Invariant Feature Transform (SIFT) [1] or Speeded Up Robust Feature (SURF) [6] identify certain pixels in the image and make a correspondence with pixels in other image to perform the corresponding task. Usually there exist variations as scale changes, rotations, deformations, occlusions, etc. between pairs of images. Feature detectors than can deal with these variations are known as invariant feature detectors. This is the case of SIFT and SURF. The price is computation time. Harris and Hessian feature detectors provide higher frame rates, but less invariance or accuracy. The approach addressed in this paper is thought as a solution encompassing different feature detectors or modes of operation on a CMOS-3D-based system. In the SIFT mode, the architecture runs the SIFT algorithm, giving high accuracy. In the Harris or Hessian modes, the accuracy is reduced in exchange of speed. The user selects the appropriate mode of operation according to the needs of the application. The feasibility of several feature detectors on a single chip is possible thanks to the fact that both SIFT and Harris- and Hessian-based feature detectors share part of the pixel-level functions.

More specifically, as a first step in SIFT, as well as Harris and Hessian algorithms in their multiscale version, the socalled Gaussian pyramid to achieve scale invariance is a must. The Gaussian pyramid comprises a set of versions of the original image (octaves) resized by a factor $1 / 4$ from one to another. Every octave is made up of a variable amount of scales. These scales are the result of the application of a Gaussian filter with a certain width $(\sigma)$ over the previous scale. The number of octaves and scales depends on the application. SIFT implements an approximation of the Laplacian operator as Difference-of-Gaussians (DoGs), which are the difference of two successive scales, conforming a pyramid of DoGs. The keypoints are found on three successive DoGs in an 8neighborhood region around one pixel of interest (27 neighbors). In the Harris- and Hessian-based methods, the keypoints are located on the scales. The SIFT algorithm is completed with three subsequent main steps: 1) the accurate location of keypoints, 2) the so-called orientation assignment, and, finally 3) the generation of a descriptor vector for every keypoint in every scale [1]. Although there is a data reduction among the main steps, e.g. from the whole image to the number of keypoints, claimed as usually less than $1 \%$ of the amount of pixels in the image, still there is a high computational burden in the higher processing stages for SIFT. This can be avoided in simpler feature detectors which do not have the stages of accurate location of keypoints, or the making of the descriptor vectors, gaining speed at the expense of less accuracy or invariance. This is the case of Harris- and Hessian-based methods. Also, apart from the Gaussian pyramid commented above, Harris- and Hessian-based methods share many pixellevel operations with SIFT. For instance, in SIFT the gradient calculation is needed for the stage of orientation assignment, 


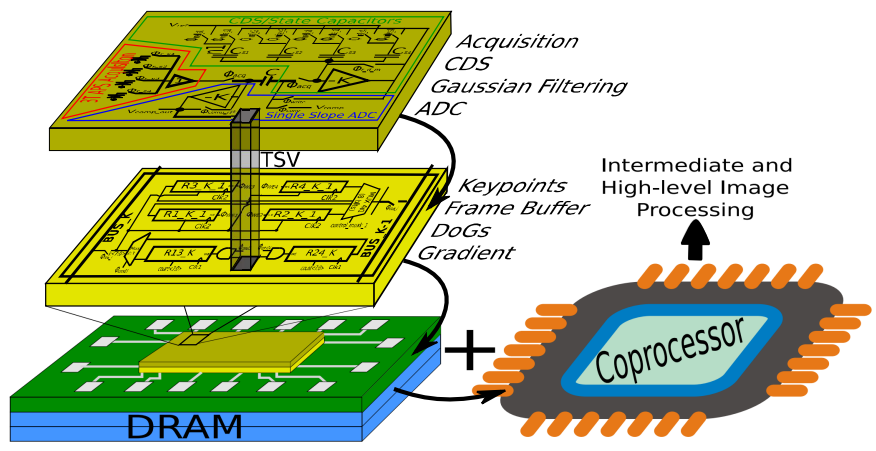

Fig. 1. CMOS-3D-based vision system. The top tier is made up of the acquisition with CDS, Gaussian pyramid generation with a switched-capacitor network and a comparator for $\mathrm{A} / \mathrm{D}$ conversion. The bottom tier contains the registers to make the conversion and store the values, as well as the hardware for the DoGs, gradient, and Harris and Hessian calculation. Both tiers are placed over a DRAM memory where the results are stored. An external coprocessor is used for control and processing tasks in higher-level stages.

which in turn is required for the extraction of the second moment matrix (or auto-correlation matrix that provides the predominant directions of the gradient in a neighborhood of a point) used in Harris. Few additional hardware modifications allow for the second derivatives for the Hessian matrix. This work takes advantage of this fact, reusing the hardware for gradient and keypoint detection for running different feature detectors. Also, the reuse of circuits combined with CMOS-3D technology permits a pixel-per-processor approach, leading to massive parallelism at pixel-level.

This paper addresses the architecture for Gaussian pyramid generation with $320 \times 240$ pixels, and a $160 \times 120$ array of analog in-pixel processors or cells, as well as the hardware to realize in parallel the SIFT, Harris and Hessian operations on a CMOS-3D stack on $130 \mathrm{~nm}$ CMOS-3D technology from Tezzaron [7]. For the time being, this technology provides two tiers tied to a foundry-provided $1 \mathrm{~Gb}$ DRAM. The Gaussian pyramid generation is provided by a Switched-Capacitor (SC) network laid in the top tier [8]. This permits to use the capacitors not only for Gaussian pyramid, but also for functions like Correlated Double Sampling (CDS) during the acquisition phase, or as analog memories at pixel-level. The registers for $\mathrm{AD}$ conversion and for the temporary storing of data and the hardware for reading and digital processing like keypoints location is placed in the bottom tier. The registers are pitch-matched with the top tier. Several approximations should be taken for the derivatives calculation by serial digitization of the four pixels. The system proposed lets run the SIFT providing keypoints, the first derivatives $(d x, d y)$, and Harris. The user could choose the best processing for the aplication. The $\sigma$-levels are user-selectable as well the number of scales. Taking a conversion time of $120 \mu s$ [10], the frame rate for processing an image with 3 octaves and six scales per octave is 180 frames/s.

\section{CMOS-3D-BASED SYSTEM}

The scheme of the CMOS-3D-based vision system is shown in Fig 1. The architecture will be implemented on a 130

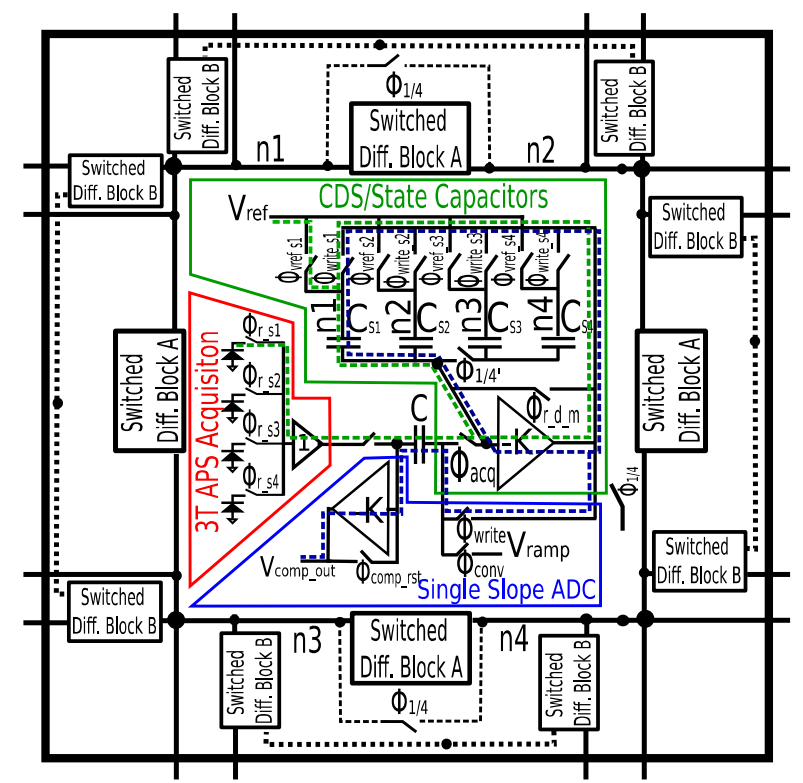

Fig. 2. Schematic of a cell in the top tier. 3T APS (red) is used along with a reusable CDS configuration that works as analog memories (green) and comparator (blue). At the same time, the analog memories are part of the switched-capacitor network which is showed around.

$n m$ CMOS-3D technology from Tezzaron [7]. Nowadays, this technology allows for two tiers. The image acquisition, the Gaussian pyramid generation, the gradient calculation, as well as the location of keypoints for Harris- and Hessianbased algorithms are provided by the CMOS-3D stack. The results are stored in a 1 Gb DRAM memory provided by Tezzaron, from where an external coprocessor can take the data to continue the processing. The external coprocessor can make low and high level processing as well as the control of the stack. An FPGA or a chip with an ARM processor would meet these goals.

\section{TOP TIER}

\section{A. Analog Processor Architecture}

Fig. 2 shows the schematic of every cell or processor located in the top tier. The different blocks are highlighted in different colors. The acquisition is performed with a 3T Active Pixel Sensor (marked in red in Fig. 2). The state capacitors that work as analog memories are enclosed in green. The comparator of the in-pixel A/D converter is indicated in blue. Finally, part of the switched-capacitor network used for the Gaussian pyramid is labeled Switched Diff. Block A and B. The state capacitors are used for the CDS and for the Gaussian pyramid. The capacitor $\mathrm{C}$ of the CDS is reused for the A/D converter. This is performed with an 8-bit single-slope A/D converter. It should be noted that we opt for a 43 T APS assignment per processor, also called cell. This is a must for a reasonable area for the massively parallel processing of our approach. At the same time, the fill-factor improves. Nevertheless, the performance decreases due to the serial acquisition and digitization of the four sensors in the first octave. A time diagram is shown in Fig. 3 for the pixel labeled $s 1\left(\phi_{r s 1}\right.$ and $C_{s 1}$ in Fig. 2) for the 


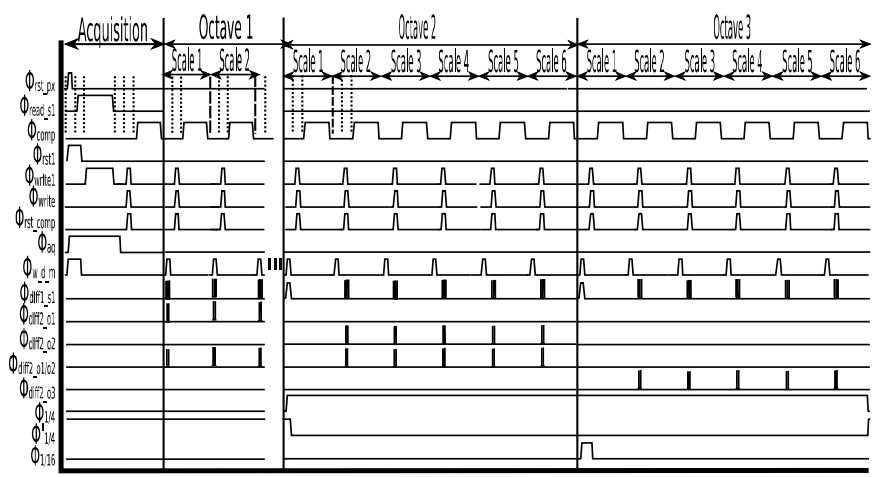

Fig. 3. Time diagram of the top tier for the pixel $s 1$. In the first octave the processing of the four pixels is in series. In the second and third octaves the four pixels are merged into only one and the processing is fully parallel.

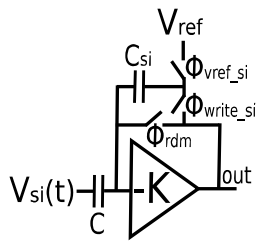

(a)

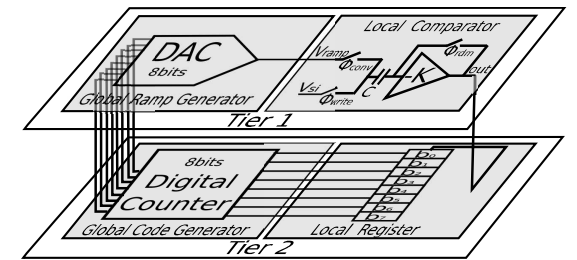

(b)
Fig. 4. (a) CDS configuration used in the top tier for the acquisition. (b) Simple scheme of the ADC shared between the two tiers.

SIFT algorithm with 3 octaves and 6 scales per octave. After the conversion, three main operations are made: Gaussian filtering, also called diffusion (signals $\phi_{\text {diff } f}$ ), copying of value in the comparator $\left(\phi_{\text {writes } 1}, \phi_{\text {write }}\right.$ and $\left.\phi_{\text {comp_rst }}\right)$, and the comparison. Note that these operations should be made in series for the four sensors.

The acquisition of the image is performed with the classical 3T-APS approach which together with the state capacitors $C_{S i}$, and the capacitor $\mathrm{C}$ make the CDS. This architecture is shown in Fig. 4(a) [9]. The result is stored in the corresponding analog memory $C_{S i}$ after the integration period, which is given by the expression:

$$
C_{V_{S i}}=V_{r e f}+\frac{C}{C_{S i}}\left[V_{S}\left(t_{0}\right)-V_{S}\left(t_{1}\right)\right]-V_{Q}
$$

where $V_{\text {ref }}$ is an analog reference signal, $V_{S}\left(t_{0}\right)$ and $V_{S}\left(t_{1}\right)$ are the values sensed at the sensor si at instants $t_{0}$ and $t_{1}$ respectively, and $V_{Q}$ is the quiescent point of the inverter.

The acquisition is controled by signals $\phi_{r_{-} s i}, \phi_{v r e f_{-} s}, \phi_{a c q}$, $\phi_{r_{-} d_{-} m}$ and $\phi_{\text {write_si }}$ with $i=1,2,3,4$. The data path is shown in Fig. 2 as a green dashed line. Disabling $\phi_{a c q}$, and enabling $\phi_{\text {write_si }}$, we can read/write the value stored at the output of the inverter as:

$$
V_{S i}=V_{r e f}+\frac{C}{C_{S i}}\left[V_{S}\left(t_{0}\right)-V_{S}\left(t_{1}\right)\right]
$$

These equations hold for a sufficiently high gain of the inverter.

The comparator for the $\mathrm{A} / \mathrm{D}$ conversion is realized with the inverter and by reusing the capacitor $C$ when the signal $\phi_{a c q}$ is turned off. The 8-bit single-slope A/D converter is

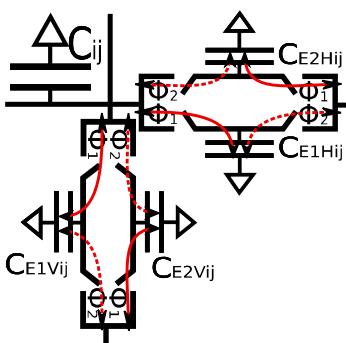

(a)

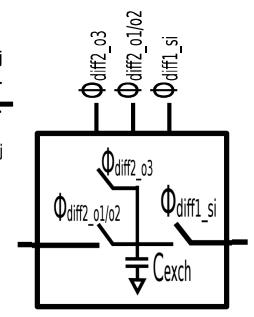

(b)

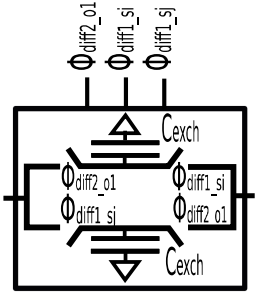

(c)
Fig. 5. Different schematis of switched-capacitor networks. (a) Simple scheme of the switched-diffusion network in a node, the ratio state/exchange Capacitors $\left(C_{i j} / C_{E}\right)$ fixes the filtering width. (b) and (c) internal structure of the Switched diff. Blok A and B.

distributed among two tiers: the analog ramp generator and the comparator in the top tier, and a register and a digital counter in the bottom tier. A scheme of such an A/D converter is displayed on Fig. 4(b). To carry out the conversion, firstly, the value given by Eq. (2) is written in the capacitor $C$, enabling signals $\phi_{\text {write_si }}, \phi_{\text {write }}$ and $\phi_{\text {comp_rst }}$. Secondly, this value is compared with the analog global ramp $\left(V_{\text {ramp }}\right)$, by enabling $\phi_{\text {conv }}$. After that, the output of the inverter is given by:

$$
V_{\text {out }}=-K\left(V_{\text {ramp }}-V_{S i}\right)+V_{Q}
$$

When the first term of Eq.(3) has a zero crossing, the comparator changes the logic value at its output. The output of the comparator is the signal that enables/disables the reading of the registers allocated in the bottom tier. This conversion signal is driven to the registers by a Through Silicon Vias (TSV). The data path of the conversion in the top tier is enclosed in a dashed blue line in Fig. 2.

The other functionality of the processors in the top tier is the Gaussian filtering, or Gaussian pyramid. This task is executed by the peripheral blocks of Fig. 2, working together with the state capacitors which again are reused. The peripheral blocks are implemented with a switched-capacitor network [8]. The switches controled by the signal $\phi_{1 / 4}$ let make the downscaling $1 / 4$ merging the value of the four state capacitors for the second octave. An in-depth explanation of the Gaussian pyramid generation is given in the next section.

\section{B. Nominal Analysis of the Diffusion Network}

The Gaussian filtering, which is needed for the Gaussian pyramid, is the solution of the heat equation. A ResistiveCapacitive (RC) Network is a natural solution of this equation. In [8] a switched-capacitor network based on a double Forward-Euler configuration was proposed. This double Forward-Euler network has the same behavior as that of a continuous-time RC network, except by the discrete exchange of charge between neighboring nodes. A scheme of a node of our switched-capacitor network is displayed on Fig.5(a). Every state capacitor is identified as $C_{i j}$, where $i, j$ are the coordinates in the network. The exchange of charge is made with the neighbors located along the cardinal directions. This 
discrete behavior lets a simple control of the $\sigma$ level by the number of cycles of the non-overlapping signals $\phi_{1}$ and $\phi_{2}$.

The Gaussian width $\sigma$ of every cycle is fixed by the relation between the state and the exchange capacitors $\left(C / C_{E}\right)$. In particular, the value of a node at a cycle $n$ is given by:

$$
\begin{aligned}
& V_{i j}(n)=V_{i j}(n-1)+\left[V_{i-1 j}(n-1)+V_{i+1 j}(n-1)+\right. \\
& \left.+V_{i j-1}(n-1)+V_{i j+1}(n-1)-4 V_{i j}(n-1)\right] \frac{\frac{C_{E}}{C}}{1+4 \frac{C_{E}}{C}}
\end{aligned}
$$

On the other hand, the value for the same node in one iteration with a discrete Gaussian kernel where only the interaction with the cardinal neighbors is considered, is modeled by Eq.(5).

$$
\begin{aligned}
& V_{i j}(n)=V_{i j}(n-1)+\left[V_{i-1 j}(n-1)+V_{i+1 j}(n-1)+\right. \\
& \left.+V_{i j-1}(n-1)+V_{i j+1}(n-1)-4 V_{i j}(n-1)\right] \frac{e^{-\frac{1}{2 \sigma^{2}}}}{1+4 e^{-\frac{1}{2 \sigma^{2}}}}
\end{aligned}
$$

By looking at equations (4) and (5), is easy to identify the $\sigma$ level per cycle as:

$$
\sigma_{0}=\left(2 \ln \frac{C}{C_{E}}\right)^{-1 / 2}
$$

The application of two successive Gaussian filters or kernels with $\sigma_{0}$ is equivalent to a Gaussian kernel with a certain $\sigma$. This property allows our hardware, which has a level of filtering $\sigma_{0}$ fixed by the $C / C_{E}$ ratio, to approach up to a certain accuracy any Gaussian kernel by recursive filtering or application of Gaussian kernels of $\sigma_{0}$. The dependence of $\sigma$ with the number of cycles $\sigma=\sigma(n)$ is given by Eq.(7).

$$
\sigma=\sqrt{\frac{2 n C_{E}}{4 C_{E}+C}}
$$

The $S$ scales of every octave for the Gaussian pyramid generation are generated with the same $S$ values of $\sigma$. The network for the generation of the first two octaves is displayed on Fig. 2. Peripheral blocks A and B make the interaction with neighbors along the cardinal directions. Fig. 5 (b) and (c) show their internal structure. As we have seen before, for every state capacitor the cell has four exchange capacitors. The endings $o 1, o 2$ and $o 3$ in the signals names denote the octaves where they are used. After executing the diffusions needed for the first octave, the four state capacitors $C_{s i}$ ( $i$ denotes the sensor) are merged into only one through the switches controled by $\phi_{1 / 4}$, making a downscaling $1 / 4$ of the image $(M / 2 \times N / 2)$. In this situation, the blocks $\mathrm{A}$ are in shortcircuit, and therefore the ratio $C / C_{E}$ increases by a factor 2 , changing the $\sigma=\sigma(n)$ relation of the system. To preserve the value of $\sigma$, the capacitors $C_{s 3}$ and $C_{s 4}$ are disabled after the merging. This is performed through the switch $\phi_{1 / 4}^{\prime}$.

Fig. 6 sketches the network structure of $16 \times 16$ pixels. For the third octave this involves one more set of switches. Those are controlled by signal $\phi_{1 / 16}$. When $\phi_{1 / 16}$ is on, the values stored in four cells are averaged in only one pixel, performing the downscaling of the original image from $M / 2 \times N / 2$ to $M / 4 \times N / 4$ resolution. After the merging, only one of the four cells keeps enable (highlighted in gray color). The filtering

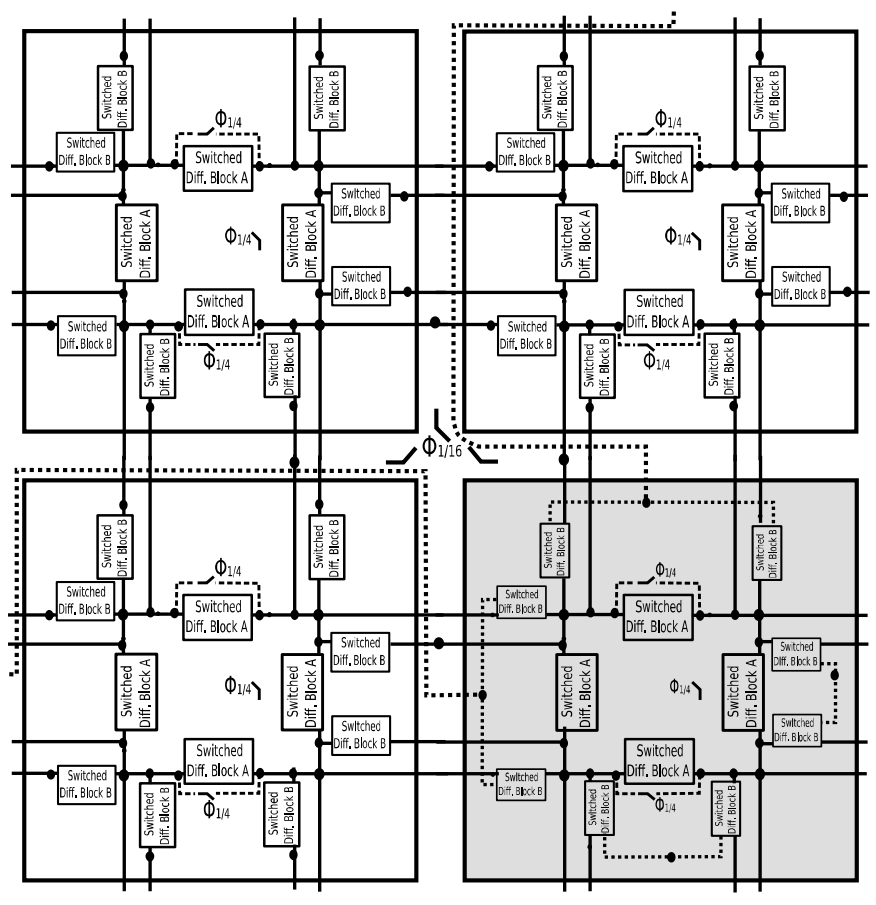

Fig. 6. Schematic of the diffusion network for a grid of $16 \times 16$ pixels of our system. In the third octave only one of the cells is used.

is made between these active cells similarly to the previous octaves through the paths drawn with a dotted-line in Fig. 6.

\section{Error Analysis in the Diffusion Network}

Several sources of error appear in the design and manufacture processes, as the non-linearity of amplifiers by the limited operating range and finite gain, the charge injection and feedthrough errors in the switches, and the mismatch in the capacitors. The main objective of this section is to make an analysis of these effects on the switched-capacitor network proposed here.

The main effect of mismatch in the switched-capacitor network is the spread of the capacitor values with respect to the nominal values $C$ and $C_{E}$. The error in the interaction of one pixel with one neighbor for a cycle $n$ is given by Eq.(8).

$$
\begin{aligned}
& \xi_{1}(n)=\frac{C_{E}}{C_{E}+C}\left[\xi_{1}(n-1)-\xi_{2}(n-1)\right]+\left[V_{1}(n-1)+\xi_{1}(n-1)\right. \\
& \left.-\mathrm{V}_{2}(n-1)-\xi_{2}(n-1)\right] \frac{1}{\left(C_{E}+C\right)^{2}} \frac{C \Delta C_{E}-C_{E} \Delta C}{1+\frac{\Delta C_{E}+\Delta C}{C_{E}+C}}
\end{aligned}
$$

$V_{1}(n-1)$ and $V_{2}(n-1)$, and $\xi_{1}(n-1)$ and $\xi_{2}(n-1)$ are, respectively, the nominal voltages and the deviation values with respect to the nominal value in the previous scale. For $n=1, \xi_{1}(0)=\xi_{2}(0)=0$. The random deviation of values along the array means that the Gaussian kernel is pixeldependant. As a result, a non isotropic diffusion might come out, having some pixels with more smoothing than others. Also, the $\sigma$ dependence with the number of cycles might change. Fig.7 shows the effect of the spread in the values of the state capacitors on the relation of $\sigma$ with the number of 

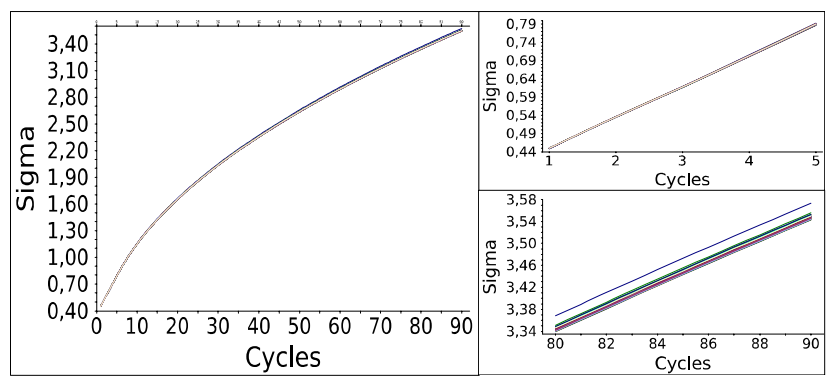

Fig. 7. Effect of the state capacitor mismatch on $\sigma=\sigma(n)$.

clock cycles $(n)$. The relation $\sigma=\sigma(n)$ is found by comparing the result of applying $n$ times a Gaussian kernel with spread with a conventional Gaussian kernel using a behavioral model in MATLAB. The $\sigma$ that minimizes the RMSE between the two images is the $\sigma$ implemented by our switched-capacitor network. Simulations of 50 random normal distributions with a standard deviation of $6 \sigma=\sqrt{C}$ were run. As seen, the effect of mismatch on the relationship $\sigma=\sigma(n)$ is really small over the whole image.

Other important error sources are the feedthrough and the charge injection from the switching. The first is due to the coupling of the control signal through the overlapping capacitance between a switch and the capacitor driven by such a switch, e.g. the switches connecting the state and the exchange capacitor. The second effect is due to the injection of the charge accumulated in the channel during inversion that goes out through the source and drain terminals when the switch turns off. As Fig.4(a) shows, in the switched-capacitor network when one switch turns off, the switch turns on at the same node. In this way, the effects of one of the switches are cancelled out by the other one in a first order consideration. Second order effects like mismatch between switches and dynamic effects appear, rendering a non-zero contribution.

\section{Area Estimate}

The cell sketched in Fig. 2 contains 4 APS, 2 inverters of gain $-K, 4$ state capacitors, an additional capacitor for offsetcancellation in the comparator used for ADC, 16 exchange capacitors, and around 60 switches. We can give an area estimate per pixel if we account for: 1) an area of $5 \mu \mathrm{m} \times 5 \mu \mathrm{m}$ per photodiode, 2) capacitors of $100 \mathrm{fF}$, which have a density of $1 \mathrm{fF} / \mathrm{\mu m}^{2}$ for the $150 \mathrm{~nm}$ CMOS-3D Tezzaron technology, 3 ) exchange capacitors of $10 \mathrm{fF}, 4)$ double-cascode inverters to enhance a high enough gain $(\mathrm{K}>60 \mathrm{~dB})$ to reduce errors in closed-loop configurations, amounting to $50 \mu \mathrm{m}^{2}$ if we take the implementation presented in [7] as a reference (FDSOI 150 $\mathrm{nm})$, 5) $2 \mu \mathrm{m}^{2}$ per switch, and 6) a TSV of $5 \mu \mathrm{m}^{2}$. All in all it yields around $250 \mu \mathrm{m}^{2}$ per pixel, which we overestimate up to $300 \mu \mathrm{m}^{2}$, accounting for the routing. These numbers would lead to $23 \mathrm{~mm}^{2}$ for an image of QVGA resolution. It should be noted that the ratio $C / C_{E}$ chosen was $100 \mathrm{fF} / 10 \mathrm{fF}$. This ratio was employed for the graph of Fig. 5, which permits a $\sigma$ range from 0.45 to 3 , enough for SIFT-based applications.

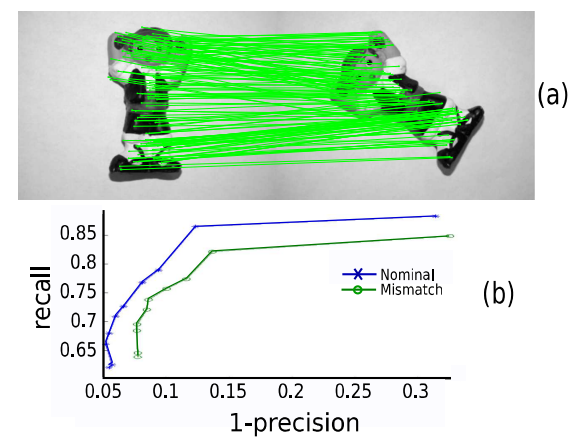

Fig. 8. (a) SIFT for object detection. (b) recall vs. 1-precision plot for object detection on the images (a).

\section{E. SIFT-based Assessment: Object Detection}

To test the validity of the switched-capacitor network and its robustness to mismatch errors, a behavioral model was implemented in MATLAB. In a first order we assume that both charge injection and feedthrough errors are canceled due to the switching mechanism of the network, as it was discussed in Section III-C. The behavior model was tested through object detection. For that we employed the SIFT implementation available at [11], assessing the so-called precision and recall for the cases of images as that shown in Fig. 8(a), in which we can see an object and its rotated version of $45^{\circ}$. The precision is defined as $p=t p /(t p+f p)$, and the recall as $r=$ $t p /(t p+f n)$, with $t p$ being the number of true positives, $f p$ the number of false positives and $f n$ the number of false negatives. $t p+f p$ is the number of matches, shown as overlapping points in Fig. 8(b). The matches are calculated by comparing the descriptor vectors of two keypoints through Euclidean distance. If this distance is below (above) a certain threshold $(t h)$, the corresponding pair of keypoints is a match. A match becomes $t p$ when it also complies with the location condition; otherwise it is a false positive. The location condition can be checked easily in a known transformation as that of Fig. 8. It is also possible to calculate $f n$. Fig. $8(\mathrm{~b})$ was obtained for several thresholds $t h$. In this case, random normal distributions with standard deviation $6 \sigma=\sqrt{C}$ were run. As it can be seen, the shape of the curve with the switched-capacitor networks subject to mismatch resembles that of the nominal one, but with little worse performance. The application dictates whether or not the mismatch is detrimental.

\section{BOtTOM TIER}

\section{A. Digital Domain Processing}

As it was said before, the A/D converter is shared among the two tiers. We opt for an 8-bit single-slope A/D converter, as it is the best option in terms of area per processor. If we distribute the comparators in the top tier, and the signals of a global counter (digital code generator) along the registers in the bottom tier, just one TSV is needed for a set of 4 pixels, namely for what we call a cell. The drawback of the singleslope $\mathrm{A} / \mathrm{D}$ converter is the long time processing, which, based on data extracted from [10], we have estimated in $120 \mu$ s per 


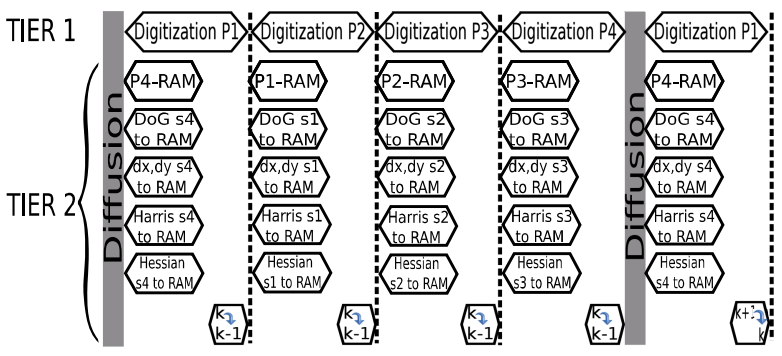

Fig. 9. Sequence of operations in the CMOS-3D stack.

conversion. The same array structure is repeated in the bottom tier, making it easier to have pitch-matched cells between the top and bottom tiers. Thus, the digitized pixels are written to an $M / 2 \times N / 2$ set of registers. Each one of these sets of registers contains 68 -bit registers. Two of them make the conversion of the scale $k$ in conjunction with the comparator of the top tier (with $k$ indicating the scale in a given octave). Two registers are needed to let the conversion of one pixel, while the others are being read for further processing. The remaining registers store the four values of the previous scale $(k-1)$. This way the whole $M \times N$ image is stored in the bottom tier. We name these 4 pixels as: $P 1, P 2, P 3$ and $P 4$, which correspond with locations $(i, j),(i, j+1),(i+1, j)$ and $(i+1, j+1)$, respectively, where $i$ is for rows and $j$ for columns. The four pixels $P 1-P 4$ are digitized in series, as there is only one Through-Silicon-Vias (TSV) per every 4pixel processor. This means that all pixels $P 1$ are digitized in one conversion cycle, $P 2$ in a second conversion cycle, and so on for pixels $P 3$ and $P 4$. Therefore, four serial conversion cycles are needed for the digitization of the whole image in the first octave.

Fig. 9 outlines the sequence of operations run in the CMOS3D stack. Fig. 10 depicts the architecture of the circuit located in the bottom tier of the CMOS-3D stack. The frame buffer is an $\mathrm{M} / 2 \times \mathrm{N} / 2$ set of registers to store the different scales of the Gaussian pyramid. After every diffusion or Gaussian filtering of the image and its digitization, the bottom tier works. Several operations are run in parallel: 1) the digitization of pixel $P 1$ at scale $k, 2$ ) scales $S(k), 3)$ difference of Gaussian between scales $k$ and $k-1$, namely $D o G(k)$, 4) horizontal gradient along the $x$ and $y$ direction for scale $k, d x(k)$ and $d y(k)$, 5) Harris and Hessian keypoint detection over scales and 6) Harris and detection Hessian over DoGs. Subsequently, the results are sorted out in groups of 128 bits ( 16 words of 8 bits each) and transfer in burst mode to the DRAM memory.

The images from the buffer array are read in groups of 20 registers (16 actual pixels +4 for windowing purposes) row by row in order to provide the 16 first and 16 second derivatives can be made at the same time. For every row $i$, the 20 columns of pixels $P i$ are selected through the multiplexers seen in Fig. 10. Four multiplexers are needed for this task. Two of them are shared by the first and the second octaves for scales $k$ and $k-1$. Both scales are required in the $D o G$ calculation. The two other multiplexers are employed for the third octave. It should be noted that for the first and the second octave,

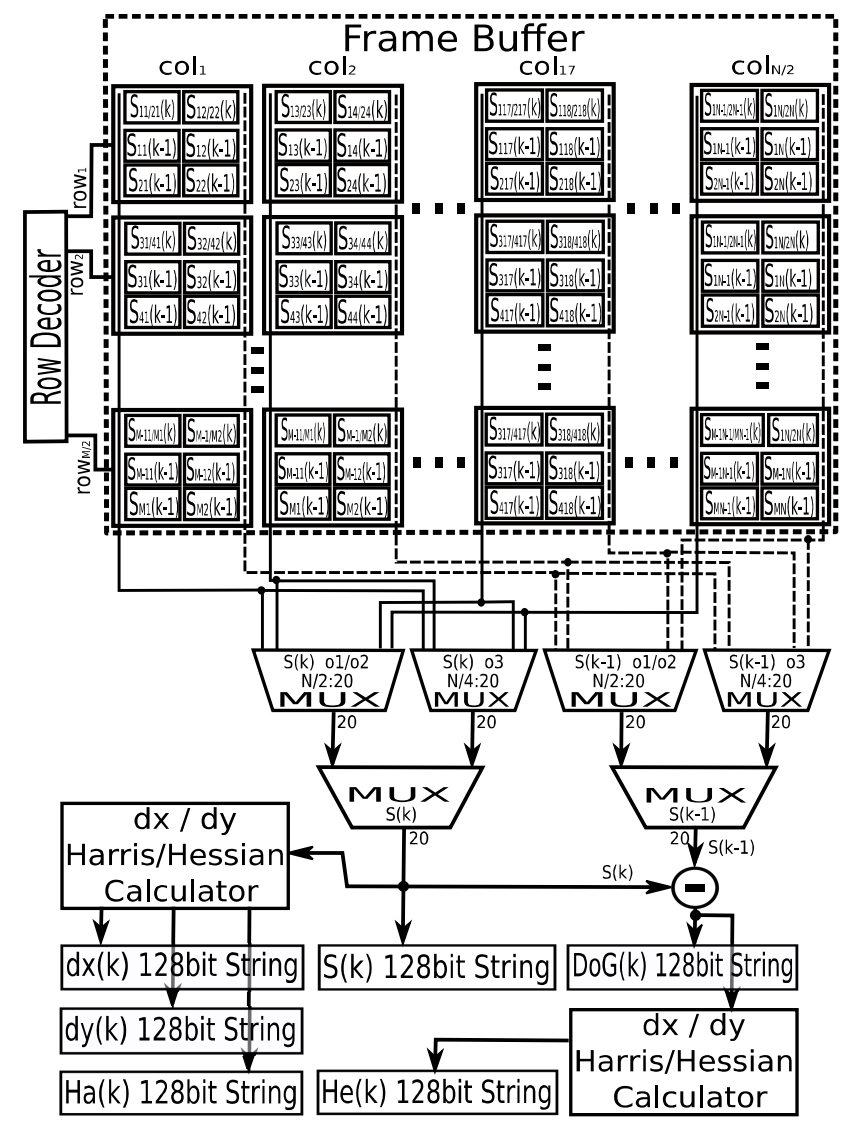

Fig. 10. Architecture of the digital circuit located in the bottom tier of the CMOS-3D stack.

the multiplexers can be shared, as we always access all the registers along a row. In the case of the first octave we need 4 "cycle" readings to transfer pixels $P 1-P 4$. In the second octave and beyond, we do such a transfer in only one cycle due to the $1 / 4$ downscaling. The lowest frequency needed for reading and doing all these operations is set by the first octave, being $10 \mathrm{Mhz}$ in order to read all the pixels $P i$ in less than $120 \mu s$, which is the time for the A/D conversion (Fig. 9).

The gradient calculation is a very common operation in image processing. Moreover, the first derivatives are used in subsequent tasks as orientation and vector descriptor of every keypoint in the SIFT algorithm, for this reason we include this functionality in our architecture. Also, the first derivatives can be used for the Harris detector. This fact permits to share hardware resources in Harris and SIFT.

In our architecture because of the assignment of 4 pixels to one processor in the top tier the reading mechanism does not permit to yield the first derivatives $d x$ and $d y$ of one pixel at one cycle. This drawback is circumvented by calculating the gradient along a different set of axes which have been rotated $45^{\circ}$ with respect to the conventional $x$ and $y$ axes. The gradient is now calculated by the next set of equations:

$$
\begin{aligned}
& d_{x}^{\prime}(i, j)=I(i+1, j+1)-I(i-1, j-1) \\
& d_{y}^{\prime}(i, j)=I(i+1, j-1)-I(i-1, j+1)
\end{aligned}
$$




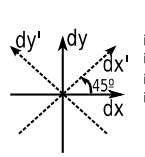

(a)

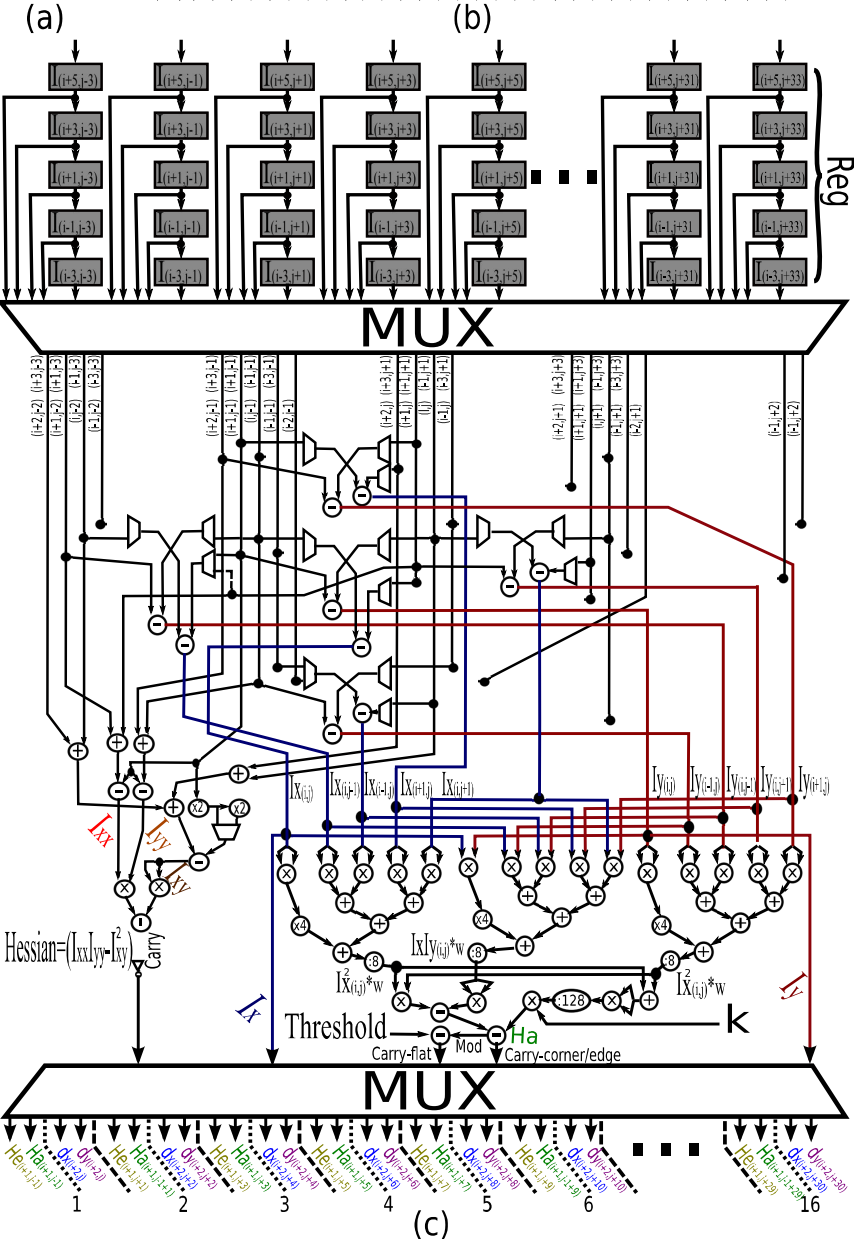

Fig. 11. Diagram block for the gradient calculation. Due to the arrangement of data, the gradient has been calculated over diagonals (conventional $x$ and $y$ axis rotated $45^{\circ}$ ) to let the Harris keypoints (a). "O" means not calculable, and " $\mathrm{X}$ " calculable, blue is the gradient and red the second derivatives (b). The 16 values of gradient, Hessian and Harris are calculated in series by the architecture shown in (c).

Fig.11 (a) illustrates this procedure. Fig.11 (b) shows the spatial arrangement of digitized values for the octave one. In the example, only the pixels $P 1$ (grey) are accessible. Fig. 11 (c) shows the block diagram for gradient, Harris and Hessian extraction. As shown in Fig. 11 (b) groups of 20 pixels are transferred row by row from the frame buffer to this block . This process is showed with an " $\mathrm{X}$ " (gradient in blue and second derivatives in red) in Fig. 11(b). The derivatives cannot be obtained at the border of the image due to the neighborhood dependance. These points are represented as "O" in the same figure. The results of the Hessian and Harris are two images of $M \times N$ sizes of 1 and 2 bits per pixel, respectively. "1" means a extrema and " 0 " is a point without significant information for SIFT. The Harris algorithm has three states: "00" is a corner, "01" an edge and " $1 X "$ a flat. The block of Fig. 11

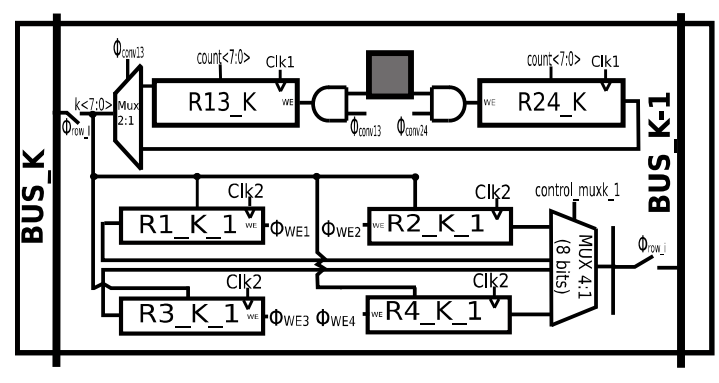

Fig. 12. A register of the frame buffer. $R 13_{-} K$ and $R 13_{-} K$ let the conversion of scale " $k$ " while k-1 is being read. The other registers store the values of the four pixels of the scale $k-1$.

makes one calculation by cycle (one pixel). The frequency of this block should be $160 \mathrm{Mhz}(16 \times 10 \mathrm{Mhz}) 16$ times higher than the reading frequency to the processing of the 16 words of a string.

Other detectors are based on the localization of characteristic points through the Hessian matrix. This matrix needs the second derivatives. The calculation of such derivatives requires the neighbors around a point in a 4-neighborhood. As it was mentioned before, in our reading mechanism, a given pixel does not have the right neighbors to perform the first derivates along the conventional $x$ and $y$ axes (horizontal and vertical directions). It would be possible, however, to do such an operation with pixels located two pixels apart. An approach to the second derivative is made by generating the neighbor located one pixel apart along the horizontal and vertical directions by interpolating the pixels located two pixels apart from the one under study. Thus, in this procedure, the neighbor at $(i+1, j)$ is generated as $I(i+1, j)=[I(i+2, j)+I(i, j)] / 2$. With this approximation the second derivatives are given by Eq.(12-13).

$$
\begin{aligned}
\mathrm{d}_{x x}(i, j) & =I(i, j+r))+I(i, j-u)-v * I(i, j) \\
\mathrm{d}_{y y}(i, j) & =I(i+u, j)+I(i, j-u))-v * I(i, j) \\
\mathrm{d}_{x y}(i, j) & =I(i+u, j+u)+I(i+u, j-u)+I(i-u, j+u)+ \\
& +I(i-u, j-u)-v * 2 * I(i, j)
\end{aligned}
$$

where $u=2$ and $v=1$ for the first octave, and $u=1$ and $v=2$ for the next octaves, given that at the second and the third octaves every pixel has the right neighbors along horizontal and vertical directions to perform the gradient along the conventional $\mathrm{x}$ and $\mathrm{y}$ axes, and thus the approach with the interpolation is not needed.

Fig. 12 displays the circuits of every set of registers in the buffer array. Every set of registers comprises 6 8-bit registers to store pixels $P 1-P 4$. As said before, there is only one TSV connecting the two tiers. This is a 1-bit signal driving two AND gates with $\phi_{c o n v 13}$ and $\phi_{c o n v 24}$ as inputs, yielding the enable signals for the top two registers, $R_{13_{-} K}$ and $R_{24_{-} K}$. The top two registers store the pixels of scale $k$. The four bottom registers keep pixels $P 1-P 4$ for scale $k-1$. Scales $k$ and $k-1$ are available on the corresponding buses of every set of registers for $D o G$ calculation. The sequence of operations to achieve every scale in the first octave is as follows. Pixel $P 1$ 
is digitized into register $R_{13_{-} K}$ with $\phi_{\text {conv13 }}$ on. Subsequently, pixel $P 2$ is digitized and stored in register $R_{24 \_}$, following a similar process with signal $\phi_{\text {conv24 }}$ on. During $\phi_{\text {conv24 }}$ on the $D o G$ for all pixels $P 1$ of scale are calculated and written into the DRAM. After the reading of pixels $P 1$ the content of register $R_{13_{-} K}$ is transferred into register $R_{1 \_K-1}$ by means of singal $\phi_{W E 1}$ on. Later on, pixels $P 3$ are digitized in register $R_{13} K$ while the pixels $P 2$ are read, and the process continues up to pixels $P 4$, completing the first octave.

\section{B. Synthetized Data}

The frequency specifications in our system are set by the operations run in parallel during the A/D conversion of a given pixel (see Fig. 9). The A/D conversion time per pixel was estimated at $120 \mu \mathrm{s}$. During this time, pixels $P i-1$, which have already been $\mathrm{AD}$-converted, are being written into the bottom DRAM. Also, the DoGs, $d x$, and $d y$ calculations and their storage in the bottom DRAM for pixels $P i-1$ are being performed at the same time as the AD-conversion of $P i$. Thus, two types of operations are run in parallel, namely, data calculation and memory-writing. The time it takes to perform both in series should be inferior to $120 \mu \mathrm{s}$.

In our design, the DoGs, $d x$ and $d y$ calculations are made in groups of 16 pixels, so for a pixel $P i$, the total number of clock cycles is given by: $(M \times N) / 16 \times 4$. In a VGA image, this renders a minimum clock frequency of $10 \mathrm{MHz}$. These numbers are not hard to achieve on a modern CMOS technology. As an example, our circuit has been synthesized on a Virtex-6 from Xilinx, reaching $375 \mathrm{MHz}$. This frequency would lead to less than $12 \mu \mathrm{s}$ for the DoGs, $d x$ and $d y$ in a VGA image. Still, $108 \mu$ s would remain for the memory storage of DoGs, $d x$ and $d y$.

The memory writing is set by the specifications of the DRAM provided by Tezzaron. In this case, the memory contains $8 \mathrm{I} / \mathrm{O}$ ports with 128 bits each. Every port supports 256 bits per cycle at $1 \mathrm{GHz}$, which yields a data transfer rate $\mathrm{TR}=256 \mathrm{Gbits} / \mathrm{s}$ at every port. For a given type of pixels $P i$ in a image $320 \times 240$ image, our system demands transfer rate of $5.6 \mathrm{Gbits} / \mathrm{s}$. As seen, the data transfer rate of the DRAM provided by Tezzaron meets the needs of our application.

Concerning area, it should be said here that a synthesis on an FPGA has been made. Nevertheless, the FPGA resources do not match naturally the implementation presented here, leading to a misleading large area occupation. Custom solutions like the one reported in [10] yield an area of $50 \mu \mathrm{m} \times 50 \mu \mathrm{m}$ for a set of 6 8-bit registers in $150 \mathrm{~nm}$ FDSOI technology, which can be taken as a basis line for the frame buffer.

\section{Conclusions}

This paper has addressed the architecture of a CMOS-3Dbased vision system for running different feature detectors. The system is thought as an approach where the user can select the most appropriate feature detector according to the needs of the application. The architecture executes two main modes: 1) SIFT mode, providing high accuracy at the cost of low speed, and 2) Harris and Hessian feature detectors, yielding speed in exchange of worse accuracy. Both modes are possible due to: 1) the CMOS-3D architecture, and 2) the fact that running SIFT implies to run some of the operations required for Harris- or Hessian-based algorithms. The work also introduces a new pixel architecture with in-pixel CDS, and in-pixel A/D conversion by means of an 8-bit single-slope A/D converter. The reuse of different circuits permits to have a reasonable area for every pixel. Also, the architecture presents an assignment of 4 3T APS per processor, rendering massively parallel processing, very adequate for operations at pixel-level, quite abundant in any feature detector. The architecture is implemented with a two tier CMOS-3D stack. The top tier contains the pixels. Every pixel is completed with the circuits needed for a switched-capacitor network. Such a network gives Gaussian filtering, needed for many feature detectors. The paper presented here has addressed a behavioral model of the top tier with manufacture errors included. The feasibility of the implementation is proven with object detection by SIFT. The bottom tier contains a frame buffer and digital circuits for further processing. The frame buffer is not only used for image storage, but it is also used for A/D conversion. An area of $300 \mu \mathrm{m}^{2}$ per pixel was estimated on the $130 \mathrm{~nm}$ CMOS-3D technology from Tezzaron. The CMOS-3D stack is tied to a $1 \mathrm{~Gb}$ DRAM provided by the foundry. The circuit addressed in this paper permits to extract the pyramid, derivatives, Harris keypoints, and the DoGs keypoints with a frame rate of 180 frames/s.

\section{ACKNOWLEDGMENT}

This work has been funded by Xunta de Galicia (Spain) and MICINN (Spain) through projects 10PXIB206037PR and TEC2009-12686.

\section{REFERENCES}

[1] D.G. Lowe, "Distinctive Image Features from Scale-Invariant Keypoints",International Journal of Computer Vision, vol. 60, no. 2, pp. 91$110,2004$.

[2] C. Harris and M. Stephens. "A combined corner and edge detector".Proceedings of the 4th Alvey Vision Conference, pp. 147151. 1988.

[3] K. Mikolajcyk and C. Schmid, "An affine invariant interest point detector".In Proceedings of the 7th European Conference on Computer Vision, Copenhagen, Denmark, vol. I, pp. 128-142, 2002.

[4] P.R. Beaudet, "Rotationally invariant image operators",Proceedings of the International Joint Conference on Pattern Recongnition, pp. 579-583, 1978.

[5] K. Mikolajcyk and C. Schmid, "Scale and affine invariant interest point detectors".International Journal of Computer Vision, vol. I, no 60, pp. 63-86, 2004.

[6] Herbert Bay, Tinne Tuytelaars, Luc Van Gool, "SURF: Speeded Up Robust Features",Proceedings of the ninth European Conference on Computer Vision, May 2006

[7] http://www.tezzaron.com.

[8] M. Suárez et al., "Switched-Capacitor Networks for Scale-Space Generation",20th European Conference on Circuit Theory and Design, pp. 189-192, Linkping, Sweden, August 29-31, 2011.

[9] Yu M Chi et al., "CMOS Camera With In-Pixel Temporal Change Detection and ADC",IEEE Journal of Solid-State Circuits, vol. 42, no. 10, pp. 2187-2196, October 2007.

[10] A. Rodríguez-Vázquez et al., "A 3D Chip Architecture for Optical Sensing and Concurrent Processing", in F. Berghmans, A. G. Mignani, C. A. van Hoof (Eds.): Optical Sensing and Detection, Proceedings of SPIE, vol. 7726, pp. 772613-1-772613-12, April 12-15, 2010

[11] http://www.eecs.umich.edu/ silvio/teaching/lectures/sift.html 